\title{
Interface habitat / forêt : enjeu et facteur de risque incendie
}

\author{
H. Belkaid ${ }^{a}$ et P. Carregab \\ UMR ESPACE, Université de Nice Sophia Antipolis, 2012, France
}

\begin{abstract}
Résumé. Les habitations, insérées au milieu de la végétation forestière, forment avec cette dernière des interfaces susceptibles de subir les conséquences de ce qu'elles peuvent provoquer comme incendies de forêt dans la région de la Kabylie, notamment sur sa partie maritime. En plus de ses caractéristiques climato-botaniques et de son aspect orographique, cette zone présente un tissu urbain diffus et éparpillé, avec plusieurs villages qui pénètrent un paysage végétal de type méditerranéen, constituant ainsi un terrain favorable à l'incendie de forêt. Dans cette région méditerranéenne qui s'étale sur une soixantaine de kilomètres, le phénomène des incendies de forêt sévit à des taux inédits par rapport à la moyenne nationale. Ce qui suscite l'intérêt de la présente étude. Le but de cette dernière est de tenter de montrer l'impact des interfaces habitat-forêt (espace vital) et de l'activité économique dominante au niveau local à savoir l'élevage (espace d'activité) sur la variabilité des incendies et sur la notion du risque. Le recensement des interfaces habitat/forêt et des unités d'élevage «statique» ou hors-sol ainsi que l'étude de leur répartition spatiale le long de la façade maritime a révélé une distribution spatiale hétérogène. La densité des interfaces et le nombre d'incendies varient considérablement d'une commune à une autre sur les cinq communes de cette région. L'étude statistique (régressions simple et multiple) a montré que la variable interface habitat/forêt contribue significativement à l'explication de la variabilité spatiale des incendies.
\end{abstract}

Les habitations, insérées au milieu de la végétation forestière, forment avec cette dernière des interfaces susceptibles de subir les conséquences de ce qu'elles peuvent, parfois, provoquer en termes d'incendies de forêt. Les activités humaines présentes dans ces interfaces constituent non seulement les enjeux principaux (enjeux économiques) mais aussi des sources potentielles de départ des feux.

Dans son plan stratégique de recherche sur les forêts méditerranéennes pour l'horizon 2010-2020, la FAO suggère de comprendre les variations socio-économiques et comportementales d'utilisation et de départ des feux tout en mettant l'accent sur l'interface habitat-forêt.

La forte concentration d'interfaces habitat-forêt dans un espace donné peut influencer la fréquence d'incendies à cause du contact permanent des habitants avec la végétation environnante. Ces zones de contact et d'interpénétration entre les espaces naturels et l'urbanisation constituent des endroits d'échange et d'exercice d'influence dans un sens comme dans l'autre (objet dynamique) d'où l'augmentation des chances d'avoir des incendies à ces endroits. En Kabylie maritime, qui est une région qui s'étale sur une soixantaine de Kilomètres au Nord de l'Algérie, où règne un climat Méditerranéen, les incendies sévissent à des taux inédits par rapport à la moyenne nationale. En plus de ses caractéristiques climato-botaniques et de son aspect orographique, cette zone présente un tissu urbain diffus et éparpillé, avec plusieurs villages qui pénètrent un paysage végétal de type Méditerranéen.

Partant du principe que l'homme est responsable de la plus grande partie des feux (Velez, 2000 ; Colin et al.,

\footnotetext{
a e-mail : hambe1007@yahoo.fr

b e-mail : pierre.carrega@unice.fr
}

2001 ; Porrero Rodriguez, 2001 in Vincent, 2005), cette étude se propose de montrer l'existence d'un rapport entre les interfaces habitat-forêt en tant que structure vitale (espace de vie de l'homme) et la fréquence des incendies de forêt, et entre la variabilité spatiale des incendies et l'activité économique dominante à savoir l'élevage hors-sol (facteurs socio-économiques).

\section{Matériels et méthodes}

L'Algérie est un pays qui connait annuellement environs 1470 incendies (Arfa \& al, 2008), qui se concentrent dans sa partie nord. La couverture végétale (toute formations confondues) perd chaque année environs 35000 ha (Meddour \& al, 2007) et le maquis gagne en moyenne 25000 ha sur la forêt (Missoumi \& al. 2002).

La Kabylie subit un préjudice inégalé en termes d'incendies de forêt par rapport au reste du territoire algérien. La partie maritime de cette région, qui présente aussi le terrain de cette étude, enregistre les nombres les plus élevés d'incendies de forêt, survenant pendant les cinq mois de la saison sèche.

Le terrain d'étude est composé de cinq communes qui sont: Mizrana, Tigzirt, Iflissen, Azeffoun et Ait Chafaâ. Elles s'étalent le long de la mer Méditerranée et forment une façade littorale, caractérisée par un relief collinaire accidenté et orienté vers la mer (Figure 2), où l'exposition Nord domine. La plupart des incendies qui se déclarent dans cette zone sont provoqués par l'homme, mais on ignore dans la majeure partie des cas quelles sont les véritables causes. Néanmoins, il est important de souligner qu'il n'existe aucun diagnostic, ni une étude d'envergure qui 


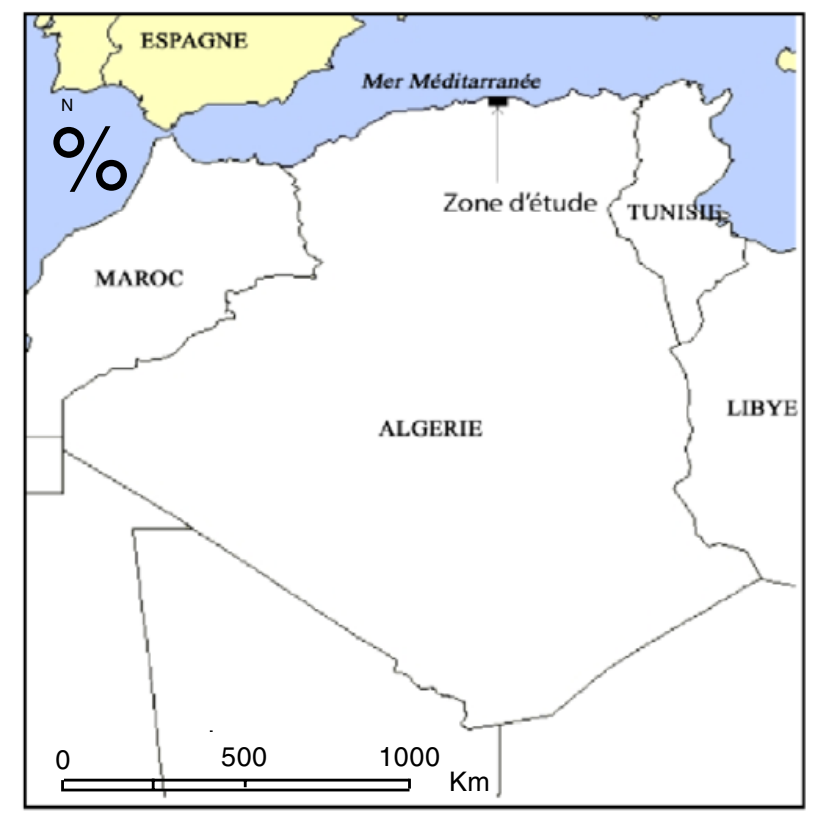

Fig. 1. Situation géographique de la zone étudiée.

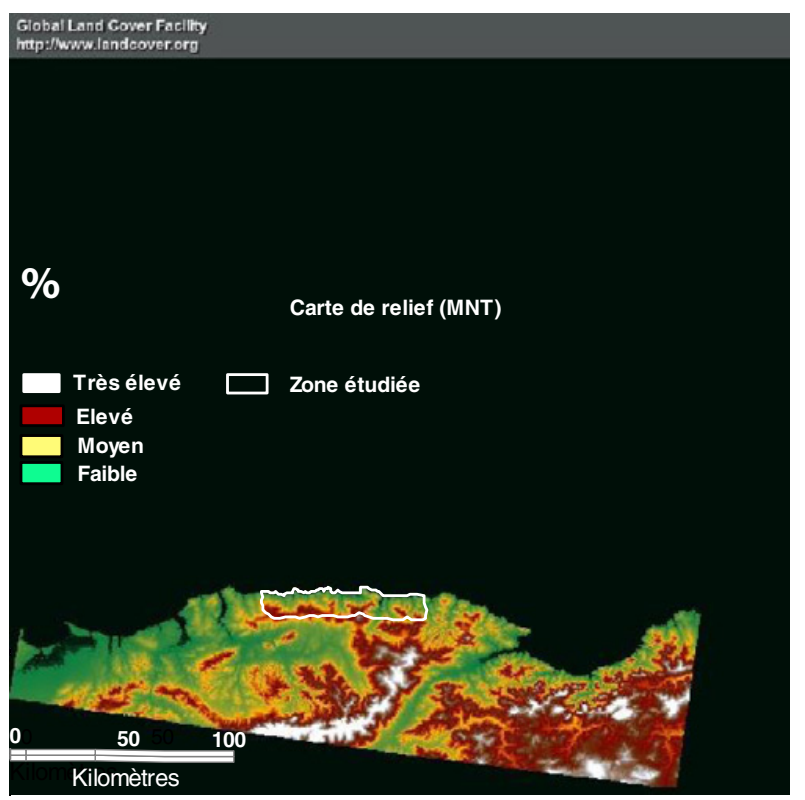

Fig. 2. Carte de relief de la Kabylie (Global Land Cover Facilities, 2012).

met en évidence l'effet de la composante humaine sur l'ampleur des incendies dans cette zone, hormis les rapports des services forestiers qui font états de bilans d'incendies. Pour la réalisation de cette étude, nous avons opté pour trois variables dont deux sont explicatives et une variable à expliquer. Il s'agit du nombre d'interfaces habitats-forêts existantes, du nombre de structures destinées à l'élevage d'animaux (élevage hors- sol) et du nombre d'incendies enregistrés pour chaque commune pour la période 19902010 .

\subsection{Les interfaces habitat-forêt}

Les interfaces habitat-forêt sont assez peu variables dans le temps, donc relativement stables pour permettre de réaliser des relevés sur des espaces différenciés (les communes) à un
Tableau 1. Matrice de corrélation.

\begin{tabular}{lccc}
\hline Variables & Interface H/F & Élevage & Incendie \\
\hline Interface H/F & $\mathbf{1 , 0 0 0}$ & 0,206 & $\mathbf{0 , 9 2 3}$ \\
Élevage & 0,206 & $\mathbf{1 , 0 0 0}$ & 0,450 \\
Incendie & $\mathbf{0 , 9 2 3}$ & 0,450 & $\mathbf{1 , 0 0 0}$ \\
\hline Les valeurs en gras sont différentes de 0 à un niveau de signifi-
\end{tabular}

cation alpha $=0,05$.

instant donné. Le repérage et le recensement exhaustif des interfaces habitat-forêt a fait ressortir des nombres précis pour chacune des cinq communes de ce territoire. Suivant la définition de l'interface habitat-forêt (Lampin \& al, 2011), il $\mathrm{n}$ y a que les maisons à vocation d'habitat situées à moins de $200 \mathrm{~m}$ des forêts, garrigues ou maquis qui sont prises en compte (Cemagref, 2010).

\subsection{Les structures d'élevages}

Ce sont des structures (légères ou lourdes) qui servent à abriter des animaux bovins, ovins, caprins et avicoles. Quelques soit le mode de l'élevage (statique ou dynamique), des structures de ce type sont mises en places pour le lieu de fixation des animaux.

Le recensement du nombre d'élevages existants dans chaque commune obéit aux mêmes critères que ceux définis dans celui des interfaces habitat-forêt. C'est-à-dire assez peu variables dans le temps et ce sont des bâtis qui se situent à moins de 200 mètres des forêts, garrigues ou maquis et ils sont réservés exclusivement aux animaux.

\subsection{Les incendies}

Durant la période 1990-2010, plusieurs incendies ont été enregistrés et répertoriés de façon cyclique et systématique par les services des forêts au niveau local. Les données sont fournies par les deux circonscriptions : la circonscription de Mizrana pour la commune de Mizrana, la commune de Tigzirt et la commune d'Iflissen et la circonscription d'Azeffoun pour la commune d'Azeffoun et la commune d'Ait Chafaâ.

$\mathrm{Au}$ total, trois variables ont été mesurées avec une relative marge d'erreur pour chacune des cinq communes afin de déterminer les effectifs et leurs variabilités intercommunales et étudier, ainsi, la nature du lien entre les variables explicatives, à savoir le nombre d'élevages et le nombre d'interfaces habitat-forêt avec la variable à expliquer, à savoir le nombre d'incendies, dans une approche statistique et analytique.

\section{Résultats et discussion}

\subsection{Test de corrélation}

Le calcul des coefficients de corrélation pour les variables considérées dans cette étude permet d'évaluer la relation qui existe entre les variables explicatives et la variable à expliquer. Le coefficient de corrélation s'écrit come suit :

$$
r(X, Y)=\frac{\operatorname{Cov}(X, Y)}{\sigma_{X} \cdot \sigma_{Y}}
$$




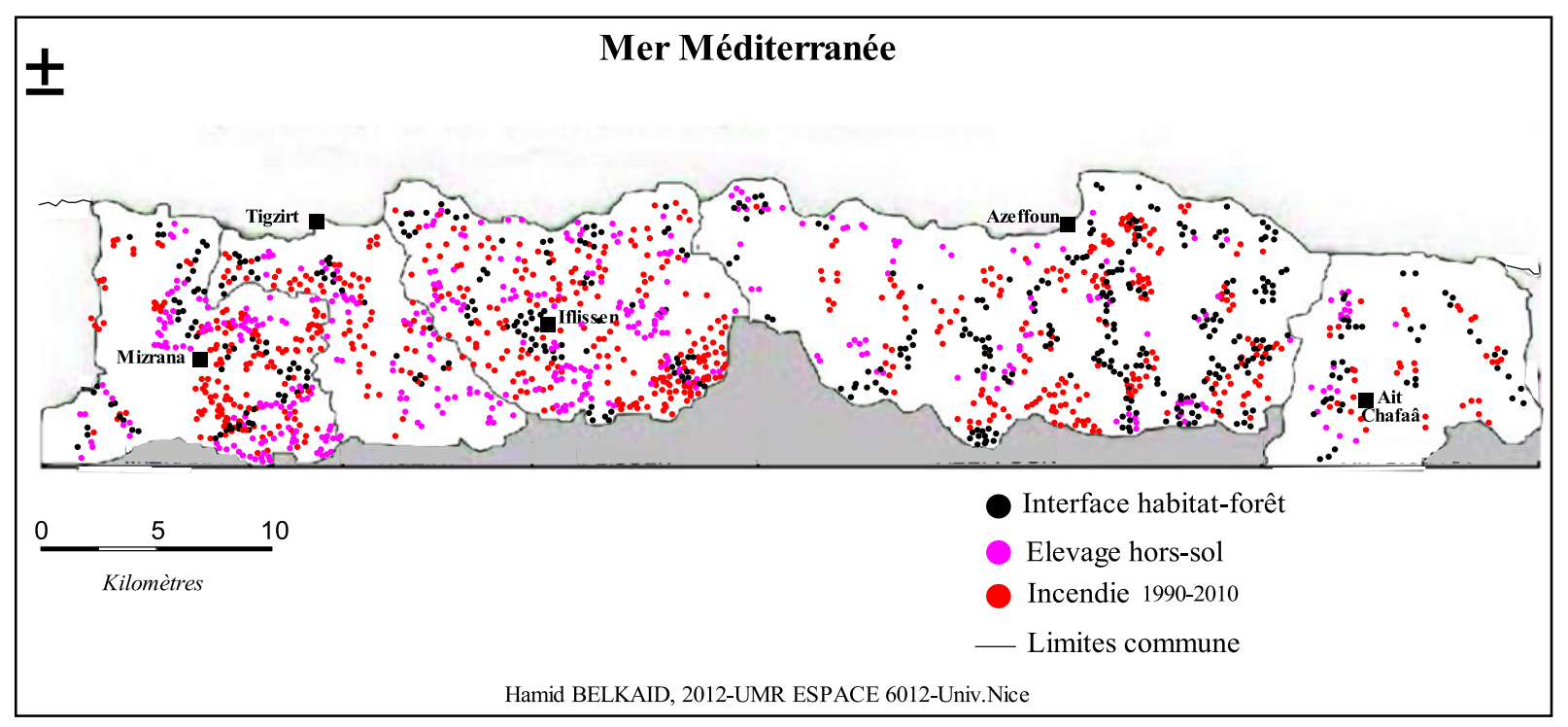

Fig. 3. Synthèse de la répartition spatiale des variables étudiées.

Tableau 2. Les p-values.

\begin{tabular}{lccc}
\hline Variables & Interface H/F & Élevage & Incendie \\
\hline Interface H/F & $\mathbf{0}$ & 0,739 & $\mathbf{0 , 0 2 5}$ \\
Élevage & 0,739 & $\mathbf{0}$ & 0,447 \\
Incendie & $\mathbf{0 , 0 2 5}$ & 0,447 & $\mathbf{0}$ \\
\hline
\end{tabular}

Où la covariance est donnée par :

$$
\operatorname{Cov}(X, Y)=\frac{1}{N} \sum_{i=1}^{N}\left(X_{i}-\bar{X}\right) \cdot\left(Y_{i}-\bar{Y}\right)
$$

[Les valeurs sont exprimées par rapport à la surface de chaque commune (densité)].

Les coefficients de corrélation de la variable incendie donnent des valeurs respectives de 0,92 (corrélation forte) et de 0,45 (corrélation faible) pour les interfaces $\mathrm{h} / \mathrm{f}$ et les élevages. La valeur du coefficient de corrélation pour les deux variables interface $\mathrm{h} / \mathrm{f}$ et élevage est de 0,2 , ceci montre qu'il n y a pas de lien, entre les deux variables explicatives (absence de redondance).

Les plus values sont données dans le tableau suivant :

Selon la valeur de p-values $(0,025)$, le risque de se tromper en rejetant l'hypothèse nulle selon laquelle les corrélations ne sont pas différentes de 0 est inférieur à 0,05 .

\subsection{Régression linéaire simple}

Compte tenu du résultat obtenu pour les coefficients de corrélation, une régression linéaire simple serait nécessaire afin de formaliser la relation entre les deux variables interfaces habitat-forêt et le nombre moyen d'incendies à l'aide d'une équation (droite de régression).

Le model linéaire s'écrit comme suite :

$$
Y=a X+b+e
$$

$Y=$ la variable à expliquer (nombre d'incendies) qui prend compte de l'existence d'autres influences autre que la variable $X$.
Tableau 3. Coefficient d'ajustement.

\begin{tabular}{lc}
\hline Observations & 5,000 \\
Somme des poids & 5,000 \\
DDL & 3,000 \\
$\mathrm{R}^{2}$ & 0,852 \\
$\mathrm{R}^{2}$ ajusté & 0,803 \\
MCE & 1651,300 \\
RMCE & 40,636 \\
MAPE & 43,679 \\
DW & 0,810 \\
\hline
\end{tabular}

$X=$ la variable explicative (interface habitat-forêt).

$a$ et $b:$ les coefficients de régression théoriques du model.

$e:$ l'erreur théorique aléatoire associée à la variable dépendante.

Intervalle de confiance : $95 \%$

Les résultats obtenus donnent un bon ajustement du model, comme le montre la valeur du coefficient de détermination $\mathrm{R}^{2}=0.85$ (Voir Tableau 3). Ceci permet également de montrer la significativité du lien entre les deux variables interfaces habitat/forêt et les incendies.

La variable interface habitat/forêt permet d'expliquer $85 \%$ de la variabilité spatiale des incendies.

Les résultats obtenus à partir de la régression linéaire simple confirme le lien entre les deux variables interface habitat/forêt et incendie, notamment celui de la droite de régression (Figure 4) où aucune des valeurs suspectes (résidus) ne se trouve en dehors de l'intervalle de confiance de $95 \%$.

Au seuil de $5 \%$, l'hypothèse $\mathrm{H}_{0}$ d'indépendance des deux variables peut être rejetée car la probabilité de se tromper en rejetant $\mathrm{H}_{0}$ est inférieur à $0,05(\mathrm{P}=0.025)$ selon le résultat obtenu (Voir Tableau 4).

\subsection{Régression linéaire multiple}

Suivant le résultat de la régression linéaire simple, nous allons tenter d'améliorer l'explication de la variabilité 

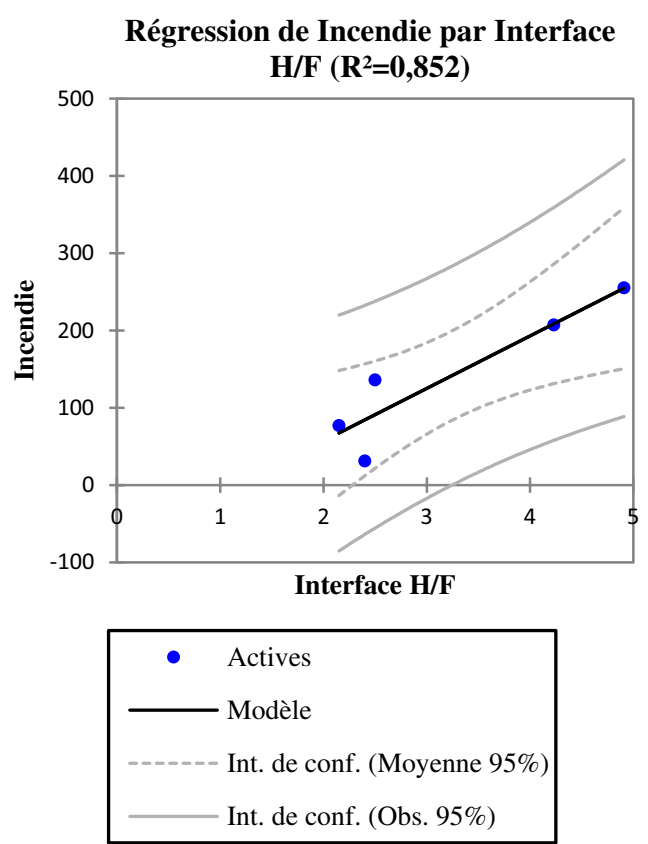

Fig. 4. Droite de régression linéaire pour les deux variables interface $\mathrm{h} / \mathrm{f}$ et l'incendie.

Tableau 4. Analyse de la variance.

\begin{tabular}{lccccc}
\hline Source & DDL & $\begin{array}{c}\text { Somme } \\
\text { des carrés }\end{array}$ & $\begin{array}{c}\text { Moyenne } \\
\text { des carrés }\end{array}$ & F & Pr $>$ F \\
\hline Modèle & 1 & 28618,900 & 28618,900 & 17,331 & 0,025 \\
Erreur & 3 & 4953,900 & 1651,300 & & \\
Total corrigé & 4 & 33572,800 & & & \\
\hline \multicolumn{7}{l}{ Calculé contre le modèle $Y=$ Moyenne $(Y)$} \\
\hline
\end{tabular}

restante en rajoutant dans le model la variable élevage pour réaliser une régression multiple.

L'espace de vie des habitants de la partie maritime de la Kabylie et l'espace d'activité dominante au niveau local, sont deux facteurs susceptibles d'influencer la variabilité des incendies dans cette localité.

$Y=f\left(X_{1}, X_{2}\right)$.

$Y=$ Variable à expliquer (nombre d'incendies).

$X_{l}=$ Variable explicative 1 (nombre d'interfaces habitatforêt).

$X_{2}=$ Variable explicative 2 (nombre d'élevage).

L'analyse de la relation entre les variables explicatives où les facteurs avec la variable à expliquer requiert la supériorité du nombre d'observations par rapport au nombre de variables considérées. Il y a pour ce cas, cinq observations et trois variables (condition satisfaite).

Le modèle linéaire multiple peut être écrit comme suit :

$Y_{i}=b_{1} X_{i 1}+b_{0}+b_{2} X_{i 2}+\ldots b_{k} X_{i k}+\varepsilon_{i} \quad$ où $i=1, \ldots, 5$

Intervalle de confiance : $95 \%$.

Le coefficient de détermination $\mathrm{R}^{2}=0,92$, ce qui montre une amélioration de l'ajustement du model après avoir rajouté la variable élevage.

La variable interface habitat-forêt et la variable élevage, prises en même temps dans l'analyse, contribuent à hauteur de $92 \%$ à expliquer la variabilité des incendies à travers les
Tableau 5. Ajustement du modèle.

\begin{tabular}{lc}
\hline Observations & 5,000 \\
Somme des poids & 5,000 \\
DDL & 2,000 \\
$\mathrm{R}^{2}$ & 0,923 \\
$\mathrm{R}^{2}$ ajusté & 0,845 \\
MCE & 1297,376 \\
RMCE & 36,019 \\
MAPE & 28,151 \\
DW & 2,535 \\
Cp & 3,000 \\
AIC & 37,259 \\
SBC & 36,087 \\
PC & 0,309 \\
\hline
\end{tabular}

cinq communes de la Kabylie maritime. Toutefois, l'activité de l'élevage pratiquée dans cette région ne semble pas avoir d'effet significatif sur les fréquences annuelles moyennes d'incendies enregistrés sur les 20 dernières années.

Ce résultat obtenu peut être amélioré en rajoutant d'autres variables comme le relief, la nature du combustible, surface agricole utile, etc.

\subsection{Analyse des résidus}

Les résidus ne suivent pas une loi normale centrée réduite $\mathrm{N}(0,1)$, donc, il y a besoin de calculer l'intervalle de confiance, qui dans ce cas donne la valeur suivante :

$$
I C=141.2 \pm 7.054 \rightarrow I C:[134.14,148.25]
$$

Plus de $95 \%$ des résidus se trouvent dans cet intervalle de confiance, ce qui confirme la linéarité du model.

\subsection{Test de significativité de Bravais-Pearson}

Étant donné que le nombre d'individus (5 observations) n'est pas assez important, il est indispensable de soumettre le test de corrélation à un test de significativité de BravaisPearson.

Selon le test de significativité de Bravais-Pearson, la valeur du coefficient de corrélation trouvé dans la table est inférieure à la valeur absolue de celui calculé pour la variable interface habitat-forêt, ce qui permet de conclure qu'il existe une relation linéaire significative entre la variable incendie de forêt et la variable interface habitatforêt dans l'espace au seuil de $5 \%$.

La valeur du coefficient de corrélation trouvé dans la table est supérieure à la valeur absolue de celui calculé pour la variable élevage, ce qui veut dire qu'il n'existe pas une relation significative entre la variable incendie et la variable élevage.

Néanmoins, ce résultat ne permet pas de conclure une relation de causalité car les interfaces habitat-forêts n'impactent pas directement l'incendie, mais çà donne une idée sur l'importance de l'interface comme élément contributif au risque incendie et ceci ne concerne que la Kabylie maritime, un modèle qui ne peut être généralisé.

Le résultat obtenu à partir de cette analyse montre qu'une part importante de la variabilité des incendies 
est expliquée par les variables interface habitat forêt et l'élevage. L'introduction de la variable élevage, a pas permis d'améliorer le résultat obtenu avec la seule variable interface habitat-forêt, mais sa contribution reste peu significative compte tenu des résultats du test de corrélation. Ceci montre également que les incendies ont plus de chance d'apparaitre dans des zones où il y a une forte présence d'interfaces habitats-forêts. Selon Cardille et $a l$, (2001) et Pew et Larsen (2001) cités dans Lampin (2009), il existe une relation très significative entre l'organisation spatiale des bâtis et la fréquence des départs de feu. Butry et al, (2002) ont affirmé que les incendies de forêt apparaissaient fréquemment dans des zones où les surfaces forestières étaient fragmentées. Par ailleurs, Mercer et Prestemon (2005) soulignent le rôle significatif des facteurs socio-économiques dans les zones fortement marquées par la présence d'interfaces habitatsforêts (Lampin, 2009).

Ces différentes affirmations sur la relation existante entre la variabilité des incendies et celle des facteurs socioéconomiques, viennent conforter le résultat de la présente étude.

\section{Références}

1. F.A.O, Plan Stratégique de Recherche sur les Forêts méditerranéennes 2010-2020, 32 (2010)
2. C. Vincent, Les feux de forêt en Méditerranée : un faux procès contre Nature, L'Espace géographique, Tome $\mathbf{3 4}$, 289-304 (2005)

3. A.M. Arfa, M.E. Benderradji et D. Altou, Les incendies de forêts en Algérie : stratégie de prévention et plan de gestion, Université Mentouri, Constantine, Algérie, 13 (2008)

4. R. Meddour, O. Meddour -Sahar et A. Derridj, Le contexte des feux de forêts dans le bassin méditerranéen, document CIHEAM, 11 (2007)

5. A. Missoumi, K. Mederbal, K. Abedelli, Apport des SIG dans la prévention et la lutte contre les incendies de forêt, Stratégie de prévention des incendies dans les forêts d'Europe du Sud, Collection Actes, Éditions P, 368 (2002)

6. C. Lampin, M. Jappiot et J.P. Ferrier, Modélisation du risque incendie de forêt dans les interfaces habitatsforêts, revue Sciences, Eaux \& Territoires, Article hors sérié n 3, 3 (2011)

7. Cemagref, guide méthodologique pour la caractérisation et la cartographie des interfaces habitat-forêt, 68 (2010)

8. C. Lampin-Maillet, Caractérisation de la relation entre organisation spatiale d'un territoire et risque d'incendie : Le cas des interfaces habitat-forêt du sud de la France, thèse de Doctorat en Géographie, Université de Provence - Aix Marseille I, 36 (2009) 